\title{
Tratamento de disfunções temporomandibulares com toxina botulínica tipo $\mathrm{A}$
}

\section{Treatment of temporomandibular disorders with botulinum toxin type $A$}

\author{
Geovana de Oliveira Polette Petrolli* \\ Pamella Monteiro Mendes** \\ Fernanda Angeloni de Souza*** \\ Matheus Coelho Blois ${ }^{* * * *}$
}

\section{Resumo}

Introdução: a disfunção temporomandibular (DTM) abrange muitos problemas clínicos nas articulações, na musculatura e em outras regiões da oroface. A origem multifatorial e sua etiologia envolvem fatores psicocomportamentais, oclusais e neuromusculares, seu diagnóstico é realizado pela anamnese detalhada, com a identificação dos fatores predisponentes, iniciadores e perpetuantes, e pelo exame físico, que consiste em palpação da musculatura, mensuração da movimentação ativa e verificação dos ruídos articulares. Objetivo: sistematizar as evidências científicas e verificar a eficácia do tratamento de disfunções temporomandibulares de origem muscular com a toxina botulínica do tipo A (TBX-A). Materiais e método: a busca bibliográfica foi realizada nas bases de dados PubMed e SciELO, com os descritores: "myofascial pain", "botulinum toxin" e "masticatory muscles". Foram analisados ensaios clínicos randomizados, que apresentaram tratamento para DTM com a utilização da TBX-A em pacientes de ambos os sexos. A qualidade metodológica dos artigos selecionados foi verificada de acordo com a escala de Jadad. Considerações finais: observou-se que o tratamento para a DTM por meio da TBX-A auxilia no tratamento de dores orofaciais permanentes como coadjuvante, aliado a tratamentos conservadores. Os estudos que demonstraram resultados clínicos significativos utilizaram uma dose total de $100 \mathrm{U}$ de TBX-A, sendo $30 \mathrm{U}$ nos músculos masseteres e $20 \mathrm{U}$ nos músculos temporais, bilateralmente.

Palavras-chave: Dor miofascial. Músculos mastigatórios. Toxina botulínica.

\section{Introdução}

A desordem ou disfunção temporomandibular (DTM) abrange problemas clínicos nas articulações, na musculatura e em outras regiões da oroface ${ }^{1}$. Segundo Dall'Magro e Valcanaia ${ }^{2}$ (2017), a DTM é um termo utilizado para descrever um grupo de condições patológicas que afetam a articulação temporomandibular (ATM), suas estruturas associadas e suas funções. A origem multifatorial e sua etiologia envolvem fatores psicocomportamentais, oclusais e neuromusculares, seu diagnóstico é realizado pela anamnese detalhada, com a identificação dos fatores predisponentes, iniciadores e perpetuantes, e pelo exame físico, que consiste em palpação da musculatura, mensuração da movimentação ativa e verificação dos ruídos articulares ${ }^{3}$.

As DTMs originárias da musculatura (miogênica) são as mais frequentes e têm como características um estado de dor crônica e sintomas específicos, como a presença de pontos gatilhos miofasciais ${ }^{4}$. Usualmente, a dor pode ser referida na região da cabeça e/ou pescoço ou mesmo estar associada a cervicalgias, cefaleias primárias e doenças reumáticas, como fibromialgia e artrite reumatoide ${ }^{5}$.

Os principais fatores etiológicos das DTMs estão relacionados a ansiedade, estresse e depressão, geralmente se manifestam em mulheres por volta da terceira década de vida. Dall'Magro e Valcanaia ${ }^{2}$ (2017) relatam que cerca de $33 \%$ da população saudável têm algum tipo de transtorno na ATM e que entre $30 \%$ e $60 \%$ apresentam algum tipo de sintomatologia, sendo que apenas $5 \%$ necessitam de tratamento.

Controlar a dor, recuperar a função do aparelho mastigatório, reeducar o paciente e amenizar cargas adversas que perpetuam o problema são os objetivos do tratamento para a $\mathrm{DTM}^{3}$. Normalmente,

Graduada em Odontologia pela Faculdade Avantis. Professora do Departamento de Prótese da Faculdade Avantis, Santa Catarina, Brasil.

Graduada em Odontologia pela Faculdade Avantis. Professora do Departamento de Prótese da Faculdade Avantis, Santa Catarina, Brasil.

Especialista em Prótese Dentária pela Universidade Federal do Rio Grande do Sul. Professora do Departamento de Prótese da Faculdade Avantis, Santa Catarina, Brasil.

*** Mestre em Implantodontia pela São Leopoldo Mandic. Professor do Departamento de Cirurgia da Faculdade Avantis, Santa Catarina, Brasil. 
os tratamentos realizados na sua fase inicial consistem em orientações, repouso, uso de placa interoclusal, fisioterapia e medidas conservadoras, como intervenção comportamental, utilização de fármacos, treinamento postural e exercícios ${ }^{5}$.

Para os pacientes que não respondem a esses tratamentos, a toxina botulínica tipo A (TBX-A) vem sendo utilizada como terapêutica. Ela promove o relaxamento dos músculos associados à mastigação, diminuindo a dor e possibilitando a função ${ }^{5}$. A toxina botulínica é uma proteína catalisadora e um relaxante muscular específico para os músculos mastigatórios, por isso foi introduzida como método terapêutico para DTM ${ }^{1}$. A TBX-A altera a intensidade da dor e as queixas relacionadas; quando associado à farmacoterapia convencional da DTM, os benefícios são potencializados ${ }^{2}$.

Discutir a utilização da toxina botulínica tipo A para tratamento de DTMs justifica-se por apresentar mais uma alternativa de tratamento na busca pelo alívio da dor e pela melhora na qualidade de vida dos portadores dessa disfunção.

É necessário compreender as causas, os sinais e as sintomas da DTM, assim como os efeitos clínicos esperados e os possíveis efeitos colaterais da toxina botulínica tipo A na terapêutica da patologia. Dessa forma, o objetivo do presente estudo foi realizar uma revisão bibliográfica para verificar e comparar as taxas de sucesso do tratamento de DTMs de origem miofascial com o uso da toxina botulínica do tipo A.

\section{Materiais e método}

Neste estudo, foram incluídos apenas ensaios clínicos randomizados que avaliaram sujeitos de ambos os sexos acometidos pelas DTMs. Os estudos deveriam contemplar intervenção por meio do tratamento com toxina botulínica tipo A e apresentar como desfecho melhora da qualidade de vida dos sujeitos avaliados. Não houve restrição quanto à faixa etária para as buscas nos bancos de dados.
A primeira busca foi realizada na base de dados PubMed, conforme os seguintes descritores: [( myofascial pain AND botulinum toxin AND masticatory muscles) OR (botulinum toxin AND mastigatory muscles AND temporomandibular joint)]; assim como os termos correspondentes na língua portuguesa: dor miofascial, toxina botulínica e músculos mastigatórios. Foi acrescido o filtro Randomized Controlled Trial. Para complementar a busca eletrônica, foi realizada a busca manual nas referências dos artigos incluídos. Inicialmente, os materiais foram selecionados de acordo com o título, em seguida, foi realizada a busca manual, os resumos foram analisados e apenas os considerados potencialmente elegíveis foram selecionados. Com base nos resumos, os artigos foram analisados na íntegra para a finalização da revisão. Em caso de divergência entre avaliadores, um terceiro avaliador tomou a decisão sobre a elegibilidade do estudo em questão.

Os ensaios clínicos randomizados foram classificados de acordo com a escala de $\mathrm{Jadad}^{6}$, que é um método para avaliar independentemente a qualidade metodológica de um ensaio clínico. Segundo The Cochrane Collaboration ${ }^{7}$, a análise da qualidade metodológica verifica-se se o desenho e a condução do estudo podem contribuir para evitar vieses, desta forma, verifica-se se a metodologia e a execução do estudo foram adequadas. Inicialmente, um total de 16 títulos e resumos foram identificados depois de uma busca eletrônica em ambas as bases de dados, utilizando a combinação específica de termos e palavras-chave. Foram identificados como relevantes dois estudos adicionais, após uma busca das listas de referências. Após a primeira fase de seleção, 12 artigos foram excluídos. As razões para a exclusão foram estudos identificados em duplicidade, com associação e descrição de outras técnicas irrelevantes para o estudo, artigos incompletos e que não apresentaram estudo randomizado. Ao final da análise, seis artigos corresponderam aos critérios de inclusão (Figura 1).

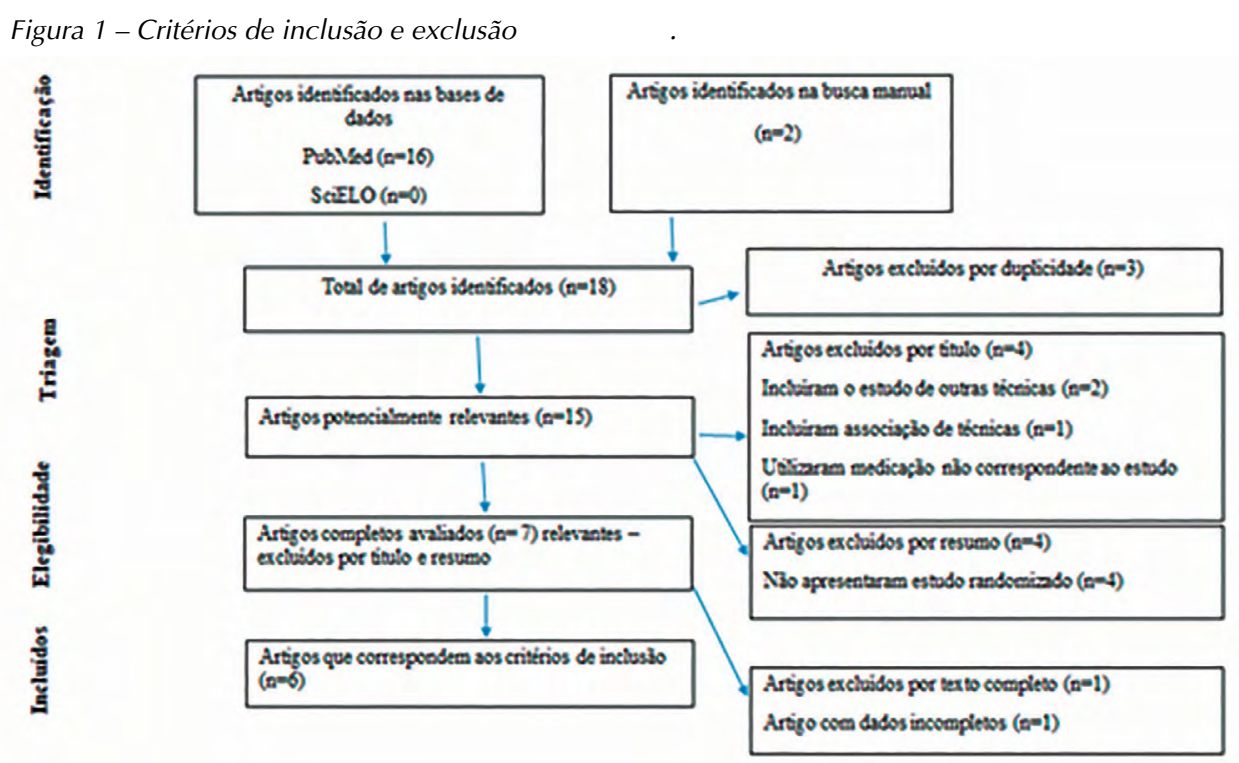

Fonte: autores. 


\section{Discussão}

Segundo a Academia Americana de Dor Orofacial, a DTM é definida como um conjunto de distúrbios que envolvem os músculos mastigatórios, a ATM e estruturas associadas ${ }^{8}$; são condições dolorosas e/ou disfuncionais, que envolvem os músculos da mastigação e/ou a ATM ${ }^{9}$. Atualmente é conhecido que a causa das DTMs é complexa e multifatorial ${ }^{9}$. Inúmeros autores destacam o papel do estresse no desencadeamento dos sintomas ${ }^{2}$. Contudo, pesquisas relatam que a etiologia da DTM está em torno da relação de três fatores: psicocomportamentais, oclusais e neuromusculares, o que mostra que as características e origens dessas alterações têm relevância no entendimento da DTM, auxiliando no planejamento e no tratamento ${ }^{3}$. As terapêuticas utilizadas têm os objetivos de controlar a dor, recuperar a função do aparelho mastigatório, reeducar o paciente e amenizar cargas adversas que perpetuem o problema ${ }^{8}$. Assim, deve ser compreendido que as próprias disfunções têm vários subgrupos, cujos tratamentos podem ser diferentes, gerando terapêuticas diferentes para o tratamento da dor, seja oclusal, ortopédica, fisiátrica, farmacológica, cirúrgica ou psicológica9 ${ }^{9}$ A etiologia indefinida, o caráter autolimitante e a altíssima eficácia recomendam a utilização inicial de terapias não invasivas e reversíveis para os pacientes que sofrem de DTM ${ }^{8}$.

Os estudos utilizaram como principal critério de inclusão as DTMs de origem miogênica. Um estudo ${ }^{10}$ selecionou pacientes com características de DTMs miogênicas e artrogênicas, ou seja, que envolvem músculos miofasciais e a ATM. O estudo de Guarda-Nardini et al. ${ }^{11}$ (2008) selecionou pacientes com DTMs de origem miofascial que apresentaram hiperatividade dos músculos mastigatórios. Apesar de todos os estudos contemplarem as disfunções de origem miofascial, também foram selecionados casos de DTMs originárias da ATM e DTMs que apresentavam como parafunção o bruxismo.

A dor miofascial é um subtipo das DTMs, e a gravidade dos sintomas varia entre dor severa ou incapacitante e limitações de movimentos ${ }^{4}$. A idade dos pacientes variou entre 16 e 53 anos, com maior concentração de pacientes na segunda e na terceira décadas de vida, corroborando a literatura mundial.

Em relação aos sujeitos das pesquisas analisadas, pode-se verificar que o sexo feminino apresenta maior incidência em dois estudos ${ }^{10,12} \mathrm{e}$ se refere à metade da população estudada em outro ${ }^{11}$. Percebe-se que as mulheres apresentam maior número nas amostras selecionadas. Segundo Sposito e Teixeira ${ }^{5}$ (2014): "A incidência das DTMs vem aumentando consideravelmente, principalmente entre mulheres de meia idade, $80 \%$ dos pacientes são do sexo feminino". Dall'Magro e Valcanaia ${ }^{2}$ (2017) afirmam que a DTM ocorre mais frequentemente na terceira década de vida, predominantemente em mulheres, na proporção 2:1.
O acompanhamento dos participantes variou de 7 dias a 6 meses. Kurtoglu et al. ${ }^{10}$ (2008) analisaram os resultados da TBX-A durante 28 dias, avaliando o efeito na atividade muscular por meio de eletromiografia, observando redução da dor e no potencial dos músculos 14 dias após a aplicação de TBX-A. Outro estudo relatou alterações na musculatura após 7 dias da aplicação de TBX-A ${ }^{11}$. Ernberg et al. $^{13}$ (2011) relatam uma redução clínica no nível de dor no primeiro mês de tratamento, mas não apresentam resultados significantes se comparado ao grupo placebo no seguimento de 3 meses. Nixdorf et al. ${ }^{12}$ (2002) avaliaram os resultados durante 3 encontros no período de 6 meses, não obtendo resultados estatisticamente significativos entre os grupos intervenção e controle.

Verifica-se que os resultados podem ser observados em um período mínimo de $7 \operatorname{dias}^{11}$. $\mathrm{O}$ acompanhamento dos resultados pelo período de 6 meses parece ser adequado para observar a recuperação neuromuscular e a diminuição dos efeitos paralisantes e analgésicos da TBX-A.

Os efeitos clínicos aparecem entre um e três dias após a administração da TBX-A, e os efeitos máximos ocorrem após uma ou duas semanas ${ }^{2}$. Mesmo que resultados possam ser verificados em um curto período, é interessante um acompanhamento maior para verificar o tempo de efetividade da TBX-A e o comportamento de redução do efeito analgésico no paciente. A recuperação da função neuromuscular pode ocorrer de três a seis semanas após a aplicação do TBX-A, pelo brotamento de novas fibras a partir do nervo original, contornando a área neuromuscular bloqueada pela toxina ${ }^{14}$.

Em relação ao tratamento, dois estudos utilizaram 100 U de TBX-A distribuídos nos músculos masseter e temporal ${ }^{10,11}$. Percebe-se que esses estudos utilizaram 3 a 4 pontos de aplicação de TBX-A nos músculos masseteres e 2 a 3 pontos nos músculos temporais, havendo redução do potencial do músculo masseter em aperto máximo, diferenças significativas na dor e estado psicológico do paciente $^{10}$, aumento dos movimentos protusivos e laterotrusivos, aumento na abertura bucal, diminuição da dor em repouso e em mastigação no grupo TBX-A, permanecendo constante no grupo controle, não houve melhora na eficiência mastigatória em nenhum dos grupos ${ }^{11}$.

Nixdorf et al. ${ }^{12}$ (2002) utilizaram 150 U de TBX-A distribuídos em 3 pontos de aplicação, nos músculos temporais e masseteres. Apesar de o grupo intervenção apresentar resultados ligeiramente melhores, não houve significância estatística entre o grupo intervenção e o placebo, apenas na amplitude menor apresentada pelos pacientes que foram submetidos a injeções de TBX-A.

O ensaio clínico de Ernberg et al. ${ }^{13}$ (2011) foi realizado apenas com aplicações de TBX-A nos músculos masseteres, relatando redução na sensação de dor superior ao grupo controle, no entanto, 
não apoiou o uso do TBX-A devido ao efeito placebo considerável. É possível que o tratamento de outros músculos miofasciais pudesse ter elevado o resultado do estudo.

Os resultados parecem ser melhores nos estudos que utilizaram $30 \mathrm{U}$ nos músculos masseteres e $20 \mathrm{U}$ nos músculos temporais ${ }^{10-15}$. Verifica-se que a abertura bucal obteve aumento no estudo de Guarda-Nardini et al. ${ }^{11}$ (2008) e redução na amplitude em outro ${ }^{12}$. Esse dado pode estar relacionado à quantidade de TBX-A utilizada, pois os dois estudos utilizaram quantidades distintas de TBX-A aplicadas nos músculos masseter e temporal. A limitação na amplitude está relacionada ao efeito paralisante e à dose de TBX-A administrada. A dose apropriada para injeção de toxina botulínica e a localização correta no músculo provocam desenervação parcial e diminuição da contratura sem paralisia completa ${ }^{16}$.

A aplicação da toxina botulínica tipo A como alternativa ao tratamento da dor miofascial tem grande efetividade, devido ao seu mecanismo de ação, pois há um bloqueio neuroquímico que inibe a liberação de acetilcolina nos terminais nervosos, levando a uma diminuição da contração muscular ${ }^{5}$.

A TBX-A provoca relaxamento muscular e, como consequência, redução da dor, permitindo ao paciente realizar movimentos e exercícios necessários para sua recuperação, durante o período de ação do TBX-A, sendo necessário intensificar o processo de reabilitação e reequilíbrio muscular com medidas físicas para obter uma melhor eficácia do tratamento ${ }^{16}$.

O relaxamento muscular causado pela TBX-A pode falhar por várias razões, como concentração insuficiente de toxina ativa injetada próximo à placa terminal motora, presença de anticorpos ou armazenamento incorreto da droga ${ }^{17}$. A avaliação da intensidade da dor nos estudos foi verificada por intermédio da escala analógica visual ${ }^{10-13}$. A aplicação dessa escala é fácil, no entanto, as avaliações e interpretações ficam sujeitas a fatores subjetivos, levando a erros de julgamento entre profissionais e pacientes.

Nixdorf et al. ${ }^{12}$ (2002) relatam a dificuldade dos pacientes em distinguir a diferença entre a intensidade da dor e o desagrado causado pela dor, fazendo com que os pacientes possam ter realizado as avaliações de formas diferentes.
Escalas para avaliação das DTMs devem ter, preferencialmente, expressões verbais que facilitem a mensuração da dor.

Dois estudos apresentaram efeitos adversos, como dor pós-aplicação, edema na região, dor à palpação e limitação de abertura bucal máxima após a aplicação na região com TBX-A. Em uma das investigações clínicas, cinco pacientes abandonaram o estudo, duas do grupo controle, por terem dores aumentadas, e três do grupo intervenção, por apresentarem paralisia do músculo zigomático maior e dor de cabeça persistente após as injeções de TBX$-\mathrm{A}^{12}$. A paralisia do músculo zigomático maior resultou em um sorriso assimétrico, o que pode ter sido causado pela difusão da TBX-A. Esse efeito adverso é transitório e reversível.

A aplicação da toxina botulínica pode causar alguns efeitos adversos e complicações decorrentes da injeção ou do produto. A maioria das adversidades é considerada leve e transitória, mas elas causam preocupação e desconforto ao paciente ${ }^{18}$. Cefaleia e náusea podem ser relatadas após a aplicação, mas tendem a ser muito leves. $O$ trauma da injeção está relacionado ao estado de ansiedade antes e/ou durante o procedimento. Esses efeitos têm regressão espontânea, mas podem ser tratados caso tragam muito desconforto; em casos raros, podem ser intensos e durar dias ${ }^{19}$.

No estudo de Ernberg et al. ${ }^{13}$ (2011), alguns pacientes relataram fraqueza muscular após as injeções de TBX-A, mas o mesmo foi observado no grupo controle. Um paciente não retornou para a segunda etapa do estudo por falta de efeito das injeções.

O estudo de Nixdorf et al. ${ }^{12}$ (2002) apresentou alto índice de perda de seguimento, de modo que se tornou difícil chegar a conclusões sólidas sobre o efeito do tratamento. Os autores relacionaram a taxa de abandono ao tratamento doloroso. A localização dos músculos através da eletromiografia por agulhamento pode ter colaborado para a sensação de dor relatada pelos pacientes, além de causar traumas como edema e hematoma e provocar alterações nos dados coletados pela eletromiografia. Indica-se a localização dos músculos por palpação, para evitar a lesão da agulha ao localizar os músculos.

As principais informações dos estudos incluídos para análise do tratamento de DTMs com a TBX-A são apresentadas no Quadro 1. 
Quadro 1 - Características metodológicas dos estudos incluídos

\begin{tabular}{|c|c|c|c|c|c|c|c|c|c|}
\hline Artigo/autoria & $\begin{array}{c}\text { Método } \\
\text { do } \\
\text { estudo }\end{array}$ & População & $\begin{array}{l}\text { Idade da } \\
\text { amostra }\end{array}$ & & Tratamento & $\begin{array}{c}\text { Avaliação/ } \\
\text { melhora } \\
\text { da dor }\end{array}$ & $\begin{array}{l}\text { Principais } \\
\text { evidências } \\
\text { encontradas }\end{array}$ & Efeitos & Formulação \\
\hline $\begin{array}{l}\text { Kurtoglu et } \\
\text { al. }{ }^{10}(2008)\end{array}$ & $\begin{array}{l}\text { Ensaio } \\
\text { clínico }\end{array}$ & $\begin{array}{c}\text { Gl: } 10 \\
\text { mulheres e } \\
2 \text { homens } \\
\text { (TBX-A) } \\
\text { GC: } 10 \\
\text { mulheres e } \\
2 \text { homens } \\
\text { (soro } \\
\text { fisiológico) } \\
\text { Total: } 24 \\
\text { pacientes }\end{array}$ & $\begin{array}{l}\text { Gl: } 16 \text { a } 53 \\
\text { anos } \\
\text { GC: } 20 \text { a } 34 \\
\text { anos }\end{array}$ & $\begin{array}{c}14 \text { e } 28 \\
\text { dias }\end{array}$ & $\begin{array}{l}\text { Gl: } 30 \cup \text { de } \\
\text { TBX-A no } \\
\text { masseter em } \\
3 \text { locais de } \\
\text { injeção e } 20 \text { U } \\
\text { no temporal } \\
\text { em } 2 \text { locais } \\
\text { de injeção } \\
\text { (bilateral) } \\
\text { GC: soro } \\
\text { fisiológico }\end{array}$ & $\begin{array}{c}\text { Escala } \\
\text { analógica } \\
\text { visual }\end{array}$ & $\begin{array}{c}\text { Redução na dor e } \\
\text { melhora do estado } \\
\text { psicológico do } \\
\text { paciente }\end{array}$ & Não & TBX-A \\
\hline $\begin{array}{l}\text { Guarda- } \\
\text { Nardini et } \\
\text { al. }{ }^{.1}(2008)\end{array}$ & $\begin{array}{l}\text { Ensaio } \\
\text { clínico }\end{array}$ & $\begin{array}{l}\text { Gl: } 10 \\
\text { pacientes } \\
\text { (TBX-A) } \\
\text { GC:10 } \\
\text { pacientes } \\
\text { (solução } \\
\text { salina) } \\
\text { Total: } 20 \\
\text { pacientes } \\
\text { (10 homens } \\
\text { e 10 } \\
\text { mulheres) }\end{array}$ & $\begin{array}{c}25 \text { a } 45 \\
\text { anos }\end{array}$ & $\begin{array}{c}1 \text { semana, } \\
1 \text { mês e } 6 \\
\text { meses }\end{array}$ & $\begin{array}{l}\text { Gl: } 30 \text { U } \\
\text { dentro do } \\
\text { músculo } \\
\text { masseter e } \\
20 \cup \text { dentro } \\
\text { da parte } \\
\text { anterior dos } \\
\text { músculo } \\
\text { temporal. } \\
\text { (Bilateral) } \\
\text { GC: soro } \\
\text { fisiológico }\end{array}$ & $\begin{array}{c}\text { Escala } \\
\text { analógica } \\
\text { visual }\end{array}$ & \begin{tabular}{|c|} 
Redução de dor \\
em repouso e na \\
mastigação no Gl, \\
permanecendo \\
constante \\
no GC. Não \\
houve melhora \\
na eficiência \\
mastigatória em \\
nenhum grupo.
\end{tabular} & Não & TBX-A \\
\hline $\begin{array}{l}\text { Nixdorf et } \\
\text { al. }{ }^{12}(2002)\end{array}$ & $\begin{array}{l}\text { Ensaio } \\
\text { clínico }\end{array}$ & $\begin{array}{l}\text { GI: TBX-A } \\
\text { GC: soro } \\
\text { fisiológico } \\
\text { (quantidade } \\
\text { de } \\
\text { pacientes } \\
\text { em cada } \\
\text { grupo não } \\
\text { relatada) } \\
\text { Total: } 15 \\
\text { pacientes } \\
\text { do sexo } \\
\text { feminino }\end{array}$ & $\begin{array}{c}18 \text { a } 45 \\
\text { anos }\end{array}$ & $\begin{array}{c}8,16 \text { e } 24 \\
\text { semanas }\end{array}$ & $\begin{array}{l}\text { Gl: } 50 \text { U } \\
\text { TBX-A em } \\
\text { cada músculo } \\
\text { masseter e } 25 \\
\text { U em cada } \\
\text { músculo } \\
\text { temporal } \\
\text { GC: soro } \\
\text { fisiológico }\end{array}$ & $\begin{array}{c}\text { Escala } \\
\text { analógica } \\
\text { visual }\end{array}$ & \begin{tabular}{|c|} 
Os resultados não \\
suportam o uso \\
de TBX-A. Não \\
houve diferença \\
estatisticamente \\
significante, \\
exceto a abertura \\
máxima, que \\
mostrou que o \\
paciente com \\
TBX-A tem \\
amplitude menor.
\end{tabular} & Sim & TBX-A \\
\hline $\begin{array}{l}\text { Ernberg et } \\
\text { al. }^{13}(2011)\end{array}$ & $\begin{array}{l}\text { Ensaio } \\
\text { clínico }\end{array}$ & $\begin{array}{l}\text { Gl: } 12 \\
\text { pacientes } \\
\text { (TBX-A) } \\
\text { GC: } 9 \\
\text { pacientes } \\
\text { (soro } \\
\text { fisiológico) } \\
\text { Total: } 21 \\
\text { pacientes } \\
\text { (não } \\
\text { informa o } \\
\text { sexo dos } \\
\text { pacientes) }\end{array}$ & (>18 anos) & $\begin{array}{c}1 \text { a } 3 \\
\text { meses }\end{array}$ & $\begin{array}{c}\text { Gl: } 50 \text { U } \\
\text { TBX-A em } \\
\text { cada masseter } \\
\text { GC: soro } \\
\text { fisiológico }\end{array}$ & $\begin{array}{c}\text { Escala } \\
\text { analógica } \\
\text { visual }\end{array}$ & $\begin{array}{c}\text { Redução } \\
\text { significativa da } \\
\text { dor (30\%) com } \\
\text { um mês após } \\
\text { a aplicação do } \\
\text { TBX-A }\end{array}$ & Sim & TBX-A \\
\hline
\end{tabular}

GC: grupo controle; GI: grupo intervenção.

Fonte: autores.

\section{Considerações finais}

Os estudos que demostraram resultados clínicos significativos utilizaram uma dose total de $100 \mathrm{U}$ de TBX-A, sendo $30 \mathrm{U}$ nos músculos masseteres e $20 \mathrm{U}$ nos músculos temporais, bilateralmente. Os efeitos benéficos puderam ser observados em sete dias, no entanto, o relaxamento dos músculos durou no máximo seis meses. Clinicamente, o uso do TBX-

-A auxilia no tratamento de dores orofaciais permanentes como coadjuvante, aliado a tratamentos conservadores. No período em que o paciente estiver sob o efeito do TBX-A, é importante intensificar o processo de reabilitação muscular. $\mathrm{O}$ custo do tratamento é alto e temporário, porém pode favorecer a intervenção de técnicas para reabilitação e intensificação do tratamento. 
Sugere-se a elaboração de mais estudos para confirmar a efetividade da TBX-A no tratamento das DTMs, com uma população maior, um seguimento mínimo de 3 meses e avaliação da dor com uma maior facilidade de compreensão pelo paciente, possibilitando identificar diferentes experiências de dor.

\section{Abstract}

Introduction: temporomandibular dysfunction (TMD) involves a number of clinical problems in joints, muscles, and other orofacial regions. The multifactorial origin and etiology involve psychobehavioral, occlusal, and neuromuscular factors. The diagnosis is performed by a detailed anamnesis with the identification of predisposing factors, initiators and perpetuants, and the physical examination, which consists of muscle palpation, measurement of the active movement, and verification of joint noises. Objective: to systematize the scientific evidence and to verify the efficacy of treatment of temporomandibular disorders of muscular origin with botulinum toxin type A (TBX-A). Materials and method: the bibliographic search was performed in the PubMed and SciELO databases, with the descriptors of "myofascial pain", "botulinum toxin", "masticatory muscles". Randomized clinical trials that presented treatment for TMD with the use of TBX-A in patients of both sexes were analyzed. The methodological quality of the articles selected was verified according to the Jadad scale. Final considerations: it was observed that treatment for TMD using TBX-A helps to treat permanent orofacial pain as a support, along with conservative treatments. The studies showing significant clinical outcomes used a total dose of $100 \cup$ of TBX-A, considering $30 \cup$ for the masseter muscles and $20 \mathrm{U}$ for the temporal muscles, bilaterally.

Keywords: Myofascial pain. Masticatory muscles. Botulinum toxin.

\section{Referências}

1. Batistello DD, Silveira AM. Disfunção temporomandibular em pacientes portadores de próteses totais superiores com redução da dimensão vertical de oclusão. J Oral Investigations 2015; 3(1):17-23.

2. Dall'Magro AK, Valcanaia T. Toxina botulínica e preenchedores na reabilitação bucomaxilofacial. São Paulo: Diilivros; 2017.

3. Sartoretto SC, Bello YD, Bona AD. Evidências científicas para o diagnóstico e tratamento da DTM e a relação com a oclusão e a ortodontia. RFO UPF 2012; 17(3):352-9.

4. Dall'Antonia M, Oliveira NRM de, Lalue SM, Antonio Sergio G. Dor miofascial dos músculos da mastigação e toxina botulínica. Rev Dor 2013; 14(1):52-7.

5. Sposito MMM, Teixeira SAF. Toxina botulínica tipo A no tratamento da dor miofascial relacionada aos músculos da mastigação: revisão sistemática. Acta Fisiatr 2014; 21(3):152-7.

6. Monteiro RNM. Metodologias de meta-análise aplicadas nas ciências da saúde [Dissertação de Mestrado]. Covilhã: Departamento de Matemática, Universidade da Beira Interior; 2010 .
7. Jadad AR, Moore RA, Carroll D, Jenkinson C, Reynolds DJ, Gavaghan DJ, et al. Assessing the quality of reports of randomized clinical trials: is blinding necessary? Elsevier Science Inc 1996; 17(1):1-12.

8. Carrara SV, Conti PCR, Barbosa JS. Termo do $1^{\circ}$ consenso em disfunção temporomandibular e dor orofacial. Dental Press J Orthod 2010; 15(3):114-20.

9. Nunes AC, Oliveira Filho AJ de, Verri FR, Santiago Júnior JF, Almeida DAF, Verri ACG. Dor orofacial. Revista Odontológica de Araçatuba 2012; 33(1):31-5.

10. Kurtoglu C, Gur OH, Kurkcu M, Sertdemir Y, Guler-Uysal F, Uysal H. Effect of botulinum toxin-A in myofascial pain patients with or without functional disc displacement. J Oral Maxillofac Surg 2008; 66(8):1644-51.

11. Guarda-Nardini L, Manfredini D, Salamone M, Salmaso L, Tonello S, Ferronato G. Efficacy of botulinum toxin in treating myofascial pain in bruxers: a controlled placebo pilot study. Cranio 2008; 26(2):126-35.

12. Nixdorf DR, Heo G, Major PW. Randomized controlled trial of botulimum toxin A for chronic myogenous orofacial pain. Pain 2002; 99(3):465-73.

13. Ernberg M, Hedenberg-Magnusson B, List T, Svensson P. Efficacy of botulinum toxin type A for treatment of persistent myofascial TMD pain: a randomized, controlled, doubleblind multicenter study. Pain 2011; 152(9):1988-96.

14. Amantéa DV, Novaes AP, Campolongo GD, Pessoa de Barros T. A utilização da toxina botulínica tipo A na dor e disfunção temporomandibular. J Bras Oclusão ATM e Dor Orofacial 2003; 3(10):170-3.

15. Lindern JJV, Neiderbagen B, Bergé S. Type A botulinum toxin in the treatment of chronic facial pain associated with masticatory hyperactivity. J Oral Maxillofac Surg 2003; 61(7):774-8.

16. Unno EK, Sakata RK, Machado IA. Estudo comparativo entre toxina botulínica e bupivacaina para infiltração de pontos-gatilho em síndrome miofascial crônica. Rev Bras Anestesiol 2005; 55(2):250-5.

17. Freud B, Schwartz M, Symington JM. Botulinum toxin: new treatment for temporomandibular disorders. J Oral Maxillofac Surg 1999; 38(5):466-71.

18. Sposito MMM. Toxina botulínica tipo A: propriedades farmacológicas e uso clínico. Acta Fisiatr 2004; 11:S7-S44.

19. Santos CS, De Mattos RM, Fulco TO. Toxina botulínica tipo A e suas complicações na estética facial. Rev Episteme Transversalis 2015; 9(2):95-106.

\section{Endereço para correspondência:}

Fernanda Angeloni de Souza

Rua Mingote Serafim, 102/902, Bairro Pioneiros 88331-025, Balneário Camboriú, SC, Brasil Telefones: (47) 997514454 - (47) 999459575 E-mail: feangelonisouza@hotmail.com

Recebido: 19/05/18. Aceito: 27/07/18. 